\title{
GENERALIZED PSEUDO-HERMITIAN OPERATORS
}

\author{
BRIAN KRITT
}

ABSTRACT. An operator in a Banach space is called generalized pseudo-hermitian if it is a generalized scalar in the sense of Foias (i.e., admits a spectral distribution) and has a real spectrum. In this paper this class of operators is characterized by the condition that the real exponential group generated by such an operator has polynomial growth in the uniform operator norm.

Introduction. An extension of the theory of spectral operators was made by Foias [1], who introduced the class of operators in Banach spaces which in lieu of spectral measures admit spectral distributions. On the other hand Kantorovitz [3] considered scalar operators in Banach space with real spectrum, which he called pseudo-hermitian. For the case of reflexive Banach spaces he characterized these operators, which are analogous to selfadjoint operators in Hilbert space, by certain analytical conditions on the real exponential group $\left\{e^{i \xi A} ; \xi \in R^{1}\right\}$ generated by such an operator. In this paper we consider the class of operators in Banach space which are generalized scalars in the sense of Foias and have real spectrum. Such operators we call generalized pseudo-hermitian (g.p.h.). We extend the results of Kantorovitz by establishing the following criterion: a bounded linear operator $A$ in a Banach space is g.p.h. if and only if the real exponential group it generates satisfies a condition

$$
\left\|e^{i \xi A}\right\| \leqq C(1+|\xi|)^{N} \quad \text { for all } \xi \in R^{1}
$$

for some positive integer $N$ and $C>0$, where \|\| denotes the operator norm. This condition is more elementary in form than those of Kantorovitz, a simplification which we attribute to the relaxation of the structural requirements on the resolution of the identity.

1. Preliminaries. By a spectral distribution (cf. [1], [4]) we mean a linear correspondence $\phi \rightarrow T_{\phi}$ between complex test functions $\phi$ from the space $D\left(R^{2}\right)$ of Schwartz and bounded linear operators in a Banach space $X$ which is continuous with respect to the usual topology of $D\left(R^{2}\right)$ and the uniform operator topology, and satisfies the additional conditions:

(a) $T_{\phi_{1} \phi_{2}}=T_{\phi_{1}} T_{\phi_{2}}$ for all $\phi_{1}, \phi_{2} \in \mathscr{D}\left(R^{2}\right)$.

Received by the editors October $1,1970$.

AMS 1970 subject classifications. Primary 47A65, 47A60, 47B15, 47B40; Secondary 46F99. 
(b) $T$ has compact support.

(c) $T_{\phi}=I$ (identity operator in $X$ ) for any test function $\phi \in D\left(R^{2}\right)$ which is identically 1 near the support of $T$.

A bounded linear operator $A$ in $X$ is said to admit the spectral distribution $T$ if $T_{\phi}=A$ for any test function $\phi \in D\left(R^{2}\right)$ which satisfies the condition $\phi(\lambda)=\lambda$ near the support of $T$. In this case we write $T_{\lambda}=A$. An operator which admits a spectral distribution is called a generalized scalar. For such an operator, Foiaş proved (Proposition 1 of [1]) the relation $\operatorname{spectrum}(A)=\operatorname{support}(T)$ for any spectral distribution $T$ which $A$ admits. As stated in the introduction, we define an operator to be generalized pseudo-hermitian (g.p.h.) if it is a generalized scalar and has a real spectrum.

We immediately present an example to show that this class of operators is nondegenerate, even in Hilbert space. Let $X$ be the Sobolev space $W^{1,2}(0,1)$ (cf. $[5$, p. 55]), and let $A$ be the operator of multiplication by the independent variable of functions in $W^{1,2}(0,1)$. Then $A$ admits the spectral distribution $T$ given by the formula $T_{\phi} f=\tilde{\phi} \cdot f$ for all $f \in W^{1,2}(0,1)$ and each $\phi \in D\left(R^{2}\right)$, where $\tilde{\phi}$ is the restriction of $\phi$ to the interval $(0,1)$. Furthermore $\operatorname{support}(T)$ $=[0,1]=\operatorname{spectrum}(A)$, so $A$ is g.p.h. But $A$ is not a scalar operator since this would imply, according to Theorem 5 of [3],

$$
\left\|e^{i \xi A}\right\| \leqq M<\infty \quad \text { for all } \xi \in R^{1}
$$

and for some $M>0$; whereas for the unit vector $f \in W^{1,2}(0,1)$ defined by $f(\xi)=1$ for all $\xi \in(0,1)$ we have the estimate

$$
\left\|e^{i \xi A}\right\| \geqq\left\|e^{i \xi A} f\right\|_{1,2}=\left\|e^{i \xi(\cdot)}\right\|_{1,2}=\left(1+|\xi|^{2}\right)^{1 / 2} .
$$

\section{Characterization of g.p.h. operators.}

Main Theorem. Let $A$ be a bounded linear operator in a Banach space $X$. Then (a) A g.p.h. implies there is a positive integer $N$ and a $C>0$ such that

$$
\left\|e^{i \xi A}\right\| \leqq C(1+|\xi|)^{N} \quad \text { for all } \xi \in R^{1} .
$$

(b) If $A$ satisfies an estimate of the form of (i), then $A$ is g.p.h.

Proof. (a) Assume $A$ admits the spectral distribution $T$ with $\operatorname{support}(T)=\operatorname{spectrum}(A) \subset R^{1}$. Then we have the decomposition $A=H+Q$, where the commuting operators $H$ and $Q$ are defined by $H=T_{\operatorname{Re} \lambda}, Q=T_{i \operatorname{Im} \lambda} . \operatorname{Re} \lambda$ and $\operatorname{Im} \lambda$ denote the real and imaginary parts of $\lambda$. Since the power series for $e^{i \xi R e \lambda}$ converges together with all derived series uniformly for $\lambda$ near support $(T)$, we have 


$$
e^{i \xi H}=T_{e^{i \xi R e \lambda}} \quad \text { for each } \xi \in R^{1} .
$$

If $K$ is a compact neighborhood of $\operatorname{support}(T)$, then there is a $C>0$ and a positive integer $N$ such that for all $\phi \in C^{\infty}\left(R^{2}\right)$ we have

$$
\left\|T_{\phi}\right\| \leqq C \sum_{|\alpha| \leq N} \sup _{K}\left|D^{\alpha} \phi\right|
$$

where $\alpha=\left(\alpha_{1}, \alpha_{2}\right)$ is a bi-index, $|\alpha|=\alpha_{1}+\alpha_{2}, D^{\alpha}=\partial^{|\alpha|} /\left(\partial x_{1}\right)^{\alpha_{1}}\left(\partial x_{2}\right)^{\alpha_{2}}$ (cf. $[2$, p. 11]). Thus we have the estimate

$$
\begin{aligned}
\left\|e^{i \xi H}\right\| & \leqq C \sum_{|\alpha| \leqslant N} \sup _{K}\left|D^{\alpha} e^{i \xi x_{1}}\right|=C \sum_{\alpha_{1}=0}^{N} \sup _{K}\left|\frac{\partial^{\alpha_{1}}}{\left(\partial x_{1}\right)^{\alpha_{1}}} e^{i \xi x_{1}}\right| \\
& =C \sum_{\alpha_{1}=0}^{N}|\xi|^{\alpha_{1}} .
\end{aligned}
$$

The function $f$ defined by $f\left(x_{1}, x_{2}\right)=f(\lambda)=\left(i x_{2}\right)^{N+1}$ vanishes, together with all derivatives of order $\leqq N$, on $R^{1}$. By an extension to the operator-valued case of Theorem 1.5 .4 of [2], we get $Q^{N+1}=T_{f}=0$. Thus

$$
\left\|e^{i \xi Q}\right\|=\left\|\sum_{h=0}^{N} \frac{(i \xi Q)^{h}}{h !}\right\| \leqq \sum_{h=0}^{N} \frac{|\xi|^{h}}{h !}\|Q\|^{h} .
$$

Combining (ii) and (iii) we get

$$
\left\|e^{i \xi A}\right\|=\left\|e^{i \xi H} e^{i \xi Q}\right\| \leqq\left\{C \sum_{\alpha_{1}=0}^{N}|\xi|^{\alpha_{1}}\right\} \cdot\left\{\sum_{h=0}^{N} \frac{|\xi|^{h}}{h !}\|Q\|^{h}\right\}
$$

from which an estimate of the form (i) is an immediate consequence.

(b) Assume $A$ satisfies the estimate (i). For each $x \in X, x^{*} \in X^{*}$ define a function $f_{x, x^{*}}$ of one complex variable by the formula

$$
f_{x, x^{*}}(\xi+i \eta)=x^{*} e^{i(\xi+i \eta) A} x .
$$

This function is entire, and, for all $\xi+i \eta$,

$$
\begin{aligned}
\left|f_{x, x^{*}}(\xi+i \eta)\right| & \leqq\left|x^{*}\right||x| C(1+|\xi|)^{N} e^{|\eta|\|\boldsymbol{A}\|} \\
& \leqq\left|x^{*}\right||x| C(1+|\xi+i \eta|)^{N} e^{|\eta|\|A\| .}
\end{aligned}
$$

By the Paley-Wiener Theorem (Theorem 1.7.7 of [2]), we conclude that $f_{x, x^{*}}$ is the Fourier-Laplace transform of a distribution $u_{x, x^{*}}$ $\in D^{\prime}\left(R^{1}\right)$ with support contained in the interval $[-\|A\|,+\|A\|]$. This means

$$
f_{x, x^{*}}(\xi+i \eta)=u_{x, x^{*}}\left(e^{-i(\xi+i \eta)(\cdot)}\right)
$$

and 


$$
u_{x, x^{*}}(\phi)=(1 / 2 \pi) \int_{R^{1}} f_{x, x^{*}}(\xi) \hat{\phi}(-\xi) d \xi
$$

for each $\phi \in \mathscr{D}\left(R^{1}\right)$, where $\hat{\phi}$ is the Fourier transform of $\phi$. From these relations is derived the following estimate:

$$
\left|u_{x, x^{*}}(\phi)\right| \leqq C / 2 \pi\left|x^{*}\right||x|\left\{\left|(1+|(\cdot)|)^{N+2} \hat{\phi}(-(\cdot))\right|_{\infty}\right\}
$$

$$
\cdot \int_{R^{1}}(1+|\xi|)^{-2} d \xi \text {. }
$$

There is a bounded linear operator $S_{\phi}$, for each $\phi \in \mathscr{D}\left(R^{1}\right)$, such that (viii)

$$
x^{*} S_{\phi} x=u_{x, x^{*}}(\phi) \quad \text { for all } x \in X, \quad x^{*} \in X^{*} .
$$

In fact, $S$ is defined by the (Bochner) integral

$$
S_{\phi}=\int_{-\infty}^{\infty} e^{i \xi A} \psi(\xi) d \xi
$$

where $\hat{\psi}=\phi$.

For $\phi \in D\left(R^{2}\right)$, define an operator $T_{\phi}$ by the formula $T_{\phi}=S_{\tilde{\phi}}$ where $\phi$ is the restriction to $R^{1}$ of the function $\phi^{\vee}$ defined by $\phi^{\sim}(\lambda)=\phi(-\lambda)$. The correspondence $\phi \rightarrow T_{\phi}$ is linear, and by (vii) and (viii) a continuous mapping of $D\left(R^{2}\right)$ into the ring of bounded operators in $X$. For $\phi_{1}, \phi_{2} \in D\left(R^{2}\right)$, and $x \in X, x^{*} \in X^{*}$, we have

$$
\begin{aligned}
& x^{*} T_{\phi_{1} \phi_{2}} x=x^{*} S_{\tilde{\phi}_{1} \tilde{\phi}_{2} x}=(1 / 2 \pi) \int_{R^{1}} x^{*} e^{i \xi A} x\left(\tilde{\phi}_{1} \cdot \tilde{\phi}_{2}\right)^{\wedge}(-\xi) d \xi \\
& =(1 / 2 \pi)^{2} \int_{R^{1}} x^{*} e^{i \xi A} x(\tilde{\phi} \hat{i} * \tilde{\phi} \hat{2})(-\xi) d \xi \\
& =(1 / 2 \pi)^{2} \int_{R^{1}} d \xi \int_{R^{1}} d \zeta\left\{x^{*} e^{i \xi A} x \tilde{\phi} \hat{i}(-\xi-\zeta) \tilde{\phi} \hat{2}(\zeta)\right\} \\
& =(1 / 2 \pi)^{2} \int_{R^{1}} d \zeta \int_{R^{1}} d \xi\left\{x^{*} e^{i \xi A} x \tilde{\phi} \hat{\imath}(-\xi-\zeta) \tilde{\phi} \hat{2}(\zeta)\right\} \\
& \text { (by the Fubini Theorem) } \\
& =(1 / 2 \pi)^{2} \int_{R^{1}} d \zeta \int_{R^{1}} d \eta\left\{x^{*} e^{-i \zeta A} e^{i \eta A} x \tilde{\phi} \hat{1}(-\eta) \tilde{\phi} \hat{2}(\zeta)\right\} \\
& \text { (where } \eta=\xi+\zeta \text { ) } \\
& =(1 / 2 \pi)^{2} \int_{R^{1}} d \zeta\left\{x^{*} e^{-i \zeta \Delta} \tilde{\phi} \hat{2}(\zeta) \int_{R^{1}} e^{i \eta \Delta} x \tilde{\phi} \hat{1}(-\eta) d \eta\right\}
\end{aligned}
$$


(since the interior in tegral exists in the sense of Bochner)

$$
\begin{aligned}
& =x^{*} T_{\phi_{2}}\left\{(1 / 2 \pi) \int_{R^{1}} e^{i \eta A} x \tilde{\phi} \hat{1}(-\eta) d \eta\right\} \\
& =x^{*} T_{\phi_{2}} T_{\phi_{1}} x .
\end{aligned}
$$

Or summarizing, $\quad T_{\phi_{2} \cdot \phi_{1}}=T_{\phi_{1} \cdot \phi_{2}}=T_{\phi_{2}} T_{\phi_{1}}$ for all $\phi_{1}, \quad \phi_{2} \in \mathcal{D}\left(R^{2}\right)$. Furthermore, support $(T)$ is contained in the real interval $[-\|A\|$, $+\|A\|]$, and if $\phi$ is a test function identically 1 on the support of $T$, then $x^{*} T_{\phi} x=u_{x, x^{*}}(\tilde{\phi})=f_{x, x^{*}}(0)=x^{*} x$ by (iv) and (v), so $T_{\phi}=I$. Hence $T$ is a spectral distribution with real support, so that it is only left to show that $A$ admits $T$. But

$$
\begin{aligned}
x^{*} A x & =-\left.i \frac{d}{d \xi} x^{*} e^{i \xi A} x\right|_{\xi=0}=-\left.i \frac{\partial}{\partial \xi} f_{x, x^{*}}\right|_{\xi=0 ; \eta=0} \\
& =\left.u_{x, x^{*}}\left(-i \frac{d}{d \xi} e^{-i \xi(\cdot)}\right)\right|_{\xi=0}=u_{x, x^{*}}(-\zeta)=u_{x, x^{*}}(\tilde{\lambda})=x^{*} T_{\lambda} x .
\end{aligned}
$$

Hence $A$ is g.p.h.

\section{Applications.}

THEOREM 2. If $A$ and $B$ are commuting g.p.h. operators in a Banach space, then $A+B$ is g.p.h.

Proof. Since $\left\|e^{i \xi(A+B)}\right\| \leqq\left\|e^{i \xi A}\right\|\left\|e^{i \xi B}\right\|$, the conclusion follows directly from the Main Theorem.

THEOREM 3. If $A$ is a g.p.h. operator in a Banach space, then so is $A^{2}$.

Proof. As in the proof of (a) of the Main Theorem, let $A=H+Q$. Then $A^{2}=H^{2}+2 H Q+Q^{2}$. Since the operators $2 H Q$ and $Q^{2}$ are nilpotent, it is enough to show that $\left\|e^{i \xi H^{2}}\right\|$ has a polynomial bound

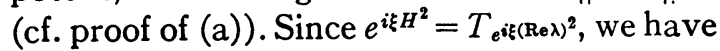

$$
\left\|e^{i \xi H^{2}}\right\| \leqq C \sum_{|\alpha| \leq N} \sup _{K}\left|D^{\alpha_{1}} e^{i \xi x_{1}^{2}}\right|=C \sum_{\alpha_{1}=0}^{N} \sup _{K}\left|\frac{\partial^{\alpha_{1}}}{\left(\partial x_{1}\right)^{\alpha_{1}}} e^{i \xi x_{1}^{2}}\right| .
$$

For each $\alpha_{1}, \sup _{K}\left|\left(\partial^{\alpha_{1}} /\left(\partial x_{1}\right)^{\alpha_{1}}\right) e^{i \xi x_{1}^{2}}\right|$ is clearly bounded by a polynomial in $|\xi|$.

TheOREM 4. If $A$ and $B$ are commuting g.p.h.operators in a Banach space, then $A B$ is g.p.h.

Proof. This follows from Theorems 2 and 3 and the relation $2 A B=(A+B)^{2}-A^{2}-B^{2}$. 


\section{REFERENCES}

1. C. Foiaş, Une application des distributions vectorielles à la théorie spectrale, Bull. Sci. Math. (2) 84 (1960), 147-158. MR 23 \#A522.

2. L. Hörmander, Linear partial differential operators, Die Grundlehren der math. Wissenschaften, Band 116, Academic Press, New York; Springer-Verlag, Berlin, 1963. MR $28 \# 4221$.

3. S. Kantorovitz, On the characterization of spectral operators, Trans. Amer. Math. Soc. 111 (1964), 152-181. MR 28 \#3329.

4. B. Kritt, Spectral decomposition of positive and positive-definite distributions of operators, Bull. Acad. Polon. Sci. Sér. Sci. Math. Astronom. Phys. 16 (1968), 865870. MR 39 \#3307.

5. K. Yosida, Functional analysis, Die Grundlehren der math. Wissenschaften, Band 123, Academic Press, New York; Springer-Verlag, Berlin, 1965. MR 31 \#5054.

UNIVERSity OF BALTIMORE, BALTIMORE, MARYLANd 21146 